\section{Systemic Lupus Erythematosus (1st Edition)}

Caroline Gordon and David Isenberg, eds. Oxford: Oxford University Press, 2016, 176 pages, $\$ 34.50$ US

Systemic Lupus Erythematosus is a small book that provides a comprehensive overview of the disease, from history and pathogenesis to treatment and management in special situations. The 9 easily read chapters and useful tables are good references. Although the book is meant to assist the practicing physician with the assessment and management of patients with systemic lupus erythematosus (SLE), only a few pages discuss conventional treatments, and much of the focus is on patients with mild disease. There are a lot of details about the epidemiology of the disease. Given the rapid changes in clinical trial results and medication targets, the chapter on biologics is already somewhat out of date, and the chapter on traditional immunosuppressive treatments, which are the mainstays of therapy for most patients with SLE, should be more detailed. The chapters on clinical features, as well as laboratory testing and investigations, are both thorough and well organized. Fortunately, the color figures on the inside covers are of much higher resolution than the black and white version in the text. The chapter on pediatric SLE mentions many differences between adult SLE and pediatric SLE, though without the specific numbers that are useful for a treating physician to know. As well, the table of autoantibodies in the chapter on pathogenesis lists antinuclear antibody as a "low sensitivity, high specificity" test, which is incorrect. This is important for anyone not experienced in treating patients with SLE, or in ordering antibody tests. Despite these concerns, Systemic Lupus Erythematosus is a concise, easily read source for more information about SLE.

JENNIFER REYNOLDS, MD, Rheumatology, University of British Columbia, Vancouver, British Columbia, Canada. Address correspondence to Dr. J. Reynolds, 3rd floor, 895 West 10th Ave., Vancouver, British Columbia V5Z 1L7, Canada.E-mail: jlstinn@hotmail.com

J Rheumatol 2016;43:8; doi:10.3899/jrheum.160674 